\title{
FORMULATION AND DEVELOPMENT OF PREBIOTIC CAPSULE
}

\section{BY}

\section{FATHIMA HAJARA ASLAM}

Thesis submitted to the university of Sri-Jayewardenapura for the award of the Degree of Master of Science in Food Science and Technology on $24^{\text {th }}$ of July 2015 


\section{Declaration}

"The work described in this thesis was carried out by me under the supervision of Dr. Jagath Wansapala and a report on this has not been submitted in whole or in part to any university or institution for another degree/diploma”.

D8. 12.2015

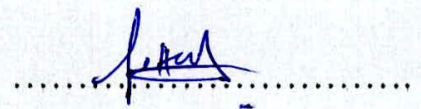

Date of submission

Signature of the Candidate 


\section{Declaration}

"I certify that the above statement made by the candidate is true and that this thesis is suitable for submission to the university for the purpose of evaluation"

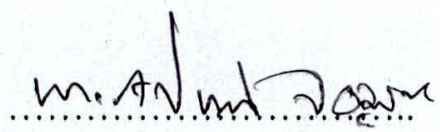

Signature of the Supervisor

Dr.Jagath Wansapala

Head of Department of Food Science and Technology

University of Sri-Jayewardenapura 


\section{CONTENTS}

\begin{tabular}{|c|c|}
\hline & Pages \\
\hline LIST OF TABLES... & .....VIII \\
\hline LIST OF FIGURES.. & ...IX \\
\hline ANEXTURE I.... & $\ldots . X$ \\
\hline ANEXTURE II............. & $\ldots X I$ \\
\hline ACKNOWLEDGEMENT & XII-XIII \\
\hline ABSTRACT... & ..XIV-XV \\
\hline
\end{tabular}

\section{CHAPTER 1: INTRODUCTION}

1.1. Background and Justification ............................................

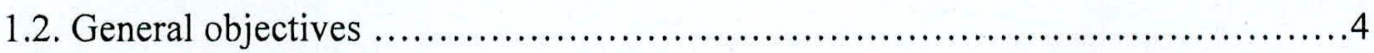

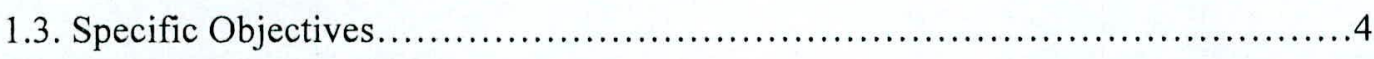

\section{CHAPTER 2: LITRETURE REVIEW}

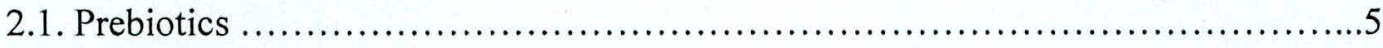

2.1.1. Properties of prebiotics..............................................6

2.1.2. Prebiotics and their availability ......................................6-7

2.2. Dietary fiber.....................................................

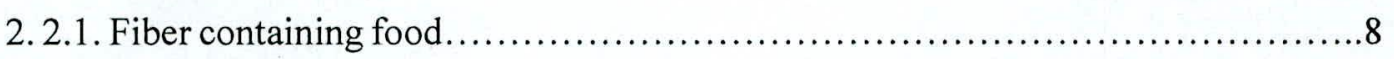

2.2.2. Fiber as prebiotics............................................... 9

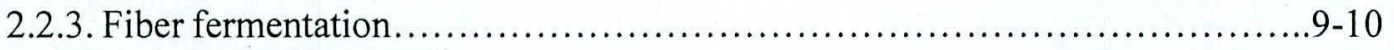


2.3. Musa sp as source of fiber 11

2.3.1. Health benefits of Musa sp............................................11

2.3.2. Musa sp pseudostem............................................. 12

2.4. Polyphenols....................................................... 13

2.4.1. Plant polyphenols............................................... 13-14

2.4.2. Classification of polyphenols ....................................... 14-16

2.4.3. Bio availability of polyphenols .....................................16-17

2.4.4. Interaction between gut microbiota and polyphenols......................17-19

2.4.1.1. Microbial metabolism of flavanones and flavonols.....................19-20

2.4.5. Dietary intake of polyphenols from plants............................20-22

2.5. Sesbania grandiflora flower as polyphenolic source.......................22-23

2.6. Non digestible oligosaccharides (NDOs) f..................................23

2.6.1. Properties of non-digestible oligosaccharides.............................24

2.6.2. Physicochemical properties of NDOs...............................25-26

2.6.3. Physiological properties of NDOs......................................26-28

2.6.4. Recommended intake of NDOs.....................................29-30

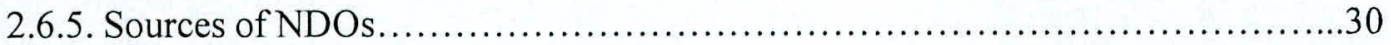

2.7. Artocarpus heterophyllus (Jack fruit) seed as NDO source......................... 31 
2.8.1. Properties of probiotics. .33

2.8.2. Health benefits of probiotics $.34-35$

2.8.3. Genus Bifidobacterium $36-38$

2.8.3.1. Bifidobacterium animalis subsp. lactis BB-12. .38

2.8.4. Genus Lactobacillus .39

2.8.4.1. Lactobacillus acidophilus $39-40$

\section{CHAPTER 3: MATERIALS AND METHODS}

3.1. Extraction of plant prebiotics....

3.1.1. Fiber extraction from Musa sp. 41

3.1.1.1. Materials 41

3.1.1.2. Method 41

3.1.2. Extraction of polyphenol extraction from Sesbania grandiflora flower .42

3.1.2.1. Materials

3.1.2.2. Method 42

3.1.3. Extraction of Non-digestible polysaccharide extraction from Artocarpous heterophyllus .43

3.1.3.1. Materials. .43

3.1.3.2. Method. 43 
3.2.1.1. Materials .44

3.2.1.2. Method. .44

3.2.2. Activation of Bifidobacterium animalis subsp. lactis BB-12.

3.2.2.2. Method .46

3.3. Determination of inoculum size of bacterial cultures 
3.5. Evaluation of growth promoting property of integrated and non-integrated prebiotic treatments on L. acidophilus .52

3.5.1. Materials .52

3.5.2. Method .52

3.6. Evaluation of growth promoting property of integrated and non-integrated prebiotic treatments on B. animalis subsp. lactis $\mathrm{BB}-12$

3.6.1. Materials .53

3.6.2. Method. .53

3.7. Determination of total polyphenol content of Sesbania grandiflora polyphenolic extract. .55

3.7.1. Materials .55

3.7.2. Method .55

3.8. Encapsulation of the prebiotic ingredients .56

3.7.1. Materials .56

3.7.2. Method. .56

\section{CHAPTER 4. RESULTS AND DISCUSSION}

4.1. The weight of extracted fiber from Musa sp pseudostem.

4.2. The weight of polyphenolic extract from Sesbania grandiflora flower.

4.3. The weight non-digestible polysaccharide extract from Artocarpus heterophyllus..57

4.4. Initial count of $L$. acidophilus and B. animalis subsp. lactis BB-12 
4.5. Total polyphenolic content in Sesbania grandiflora flower polyphenolic extract...58

4.6. Growth stimulation caused by integrated prebiotic treatment toward L. acidophilus

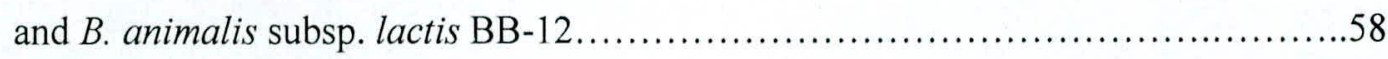

4.7. Growth stimulation caused by non- integrated prebiotic treatment toward $L$.

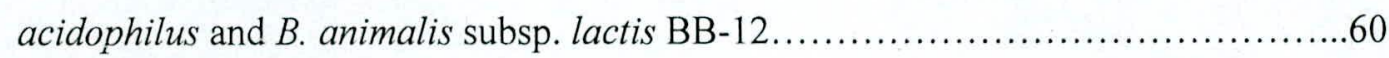

4.8. Composition of prebiotic capsule 60

4.9. Prebiotic potential of plant extracts. 61

4.9.1. Prebiotic potential of Musa sp fiber isolates .62

4.9.2. Prebiotic potential of polyphenolic extracts of $S$. grandiflora flower petals...63

4.8.3. Prebiotic potential of non-digestible polysaccharide extracts of $A$. heterophyllus seed. .64

4.10. The impact of integrated prebiotic treatment combination on L. acidophilus...6465

4.11. The impact of integrated prebiotic treatment combination on B. animalis subsp. lactis $\mathrm{BB}-12$ .65

4.12. The impact of non-integrated prebiotic treatments on L. acidophilus .67

4.13. The impact of non-integrated prebiotic treatment combination on B.animalis subsp. lactis BB-12 $67-68$ 


\section{CHAPTER 5: CONCLUSION}

5.1. Effect of integrated prebiotic treatments compared to non-integrated prebiotic treatments. .69

5.1.1. Effect of integrated prebiotic treatments on L. acidophilus and B. animalis subsp. lactis BB-12 .69

5.1.2. The effect of Non-integrated prebiotic treatments on test probiotics. .70

RECOMMENDATION FOR FURTHER WORK .70

REFERENCES $71-81$ 


\section{LIST OF TABLES}

Table 2.1. Proximate composition of Musa sp pseudostem........................12

Table 2.2. Total dietary fiber content of Musa sp pseudostem.......................12

Table 3.1. The weight of prebiotic extracts combined in formulating treatments........51

Table 3.2. Prebiotic treatments combination designed and the composition in weight/volume........................................................... 51

Table 4.1 Initial bacterial counts of inoculum in CFU/mL..........................57

Table 4.2. Growth of probiotic species with integrated prebiotic treatments...........58

Table 4.3. Growth of probiotic species with non-integrated prebiotic treatments........60 


\section{LIST OF FIGURES}

Figure 2.1. Classification and chemical structures of major polyphenols.

Figure 3.1 Activation of lyophilized bacterial cultures. .46

Figure 3.2. Appearance of activated lyophilized test cultures.... .46

Figure 3.3. Growth of the bacterial cultures in the presence of integrated prebiotic treatments

Figure.4.1. L. acidophilus colony grown with Treatment B compared with control....59

Figure 4.2. B. animalis subsp. lactis BB-12 colony grown with Treatment D compared with control .59

Figure 4.3. The effect imposed by integrated prebiotic treatments on L.acidophilus and B. animalis subsp. lactis $\mathrm{BB}-12$. .66

Figure 4.4. The effect imposed by non-integrated prebiotic treatments on L. acidophilus and B. animalis subsp. lactis $\mathrm{BB}-12$. .68 


\section{ANEXTURE I}

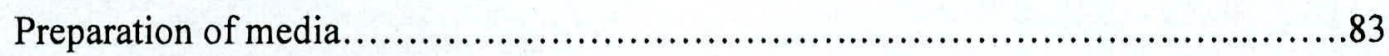

Preparation of MRS agar..................................................... 83

Preparation of MRS L-cysteine agar $\mathrm{HCl}$ agar...................................83

Sterilizing microbiological media and other equipment..........................8 
ANEXTURE II

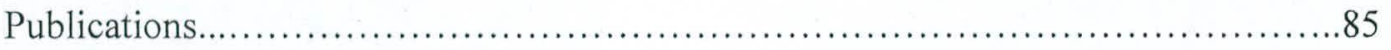

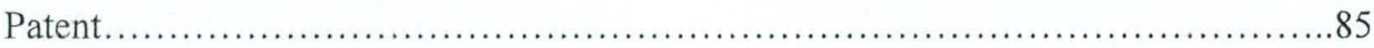




\begin{abstract}
AKNOWLEDGEMENT
This is to express my deep sense of gratitude to all those, who helped me to complete this research project successfully. I would like to express my sincere gratitude and heartfelt thanks to my supervisor Dr. Jagath Wansapala, Head of department of Food Science and Technology and coordinator for Food science and Technology program, University of Sri-Jayewardenepura for his irreplaceable generosity, inspiring, guidance and encouragement made me successful on this work.

I wish to express my sincere thanks to the Managing Director of MicroChem Laboratories Manel Perera and other staff members for their interest and valuable assistance toward this research project.

I am grateful for Samanthi Udayangani and Anusha Kaneshapillai who gave massive support in completion of this project.

Countless thanks goes to Dr. Rupika Perera, Assistant coordinator for Food Science and Technology programme from Department of Food Science and Technology University of Sri-Jayewardenepura, for her assistance and generous support during the laboratory session of this research project.

I also wish to thanks all the members of the laboratory of the Food Science and Technology for their kind cooperation and warm friendship.

I greatly appreciate Mr. Dias, Senior Lecturer of the Statistic Department from University of Sri-Jayewardenepura for his immense support in statistical analysis of the data obtained.
\end{abstract}


I would like to take this opportunity to express my sincere thanks to Mr. Ahamed Aslam and Abdus Sammad Ibrahim who funded this project. Last but not least support goes to Ms. Mufeeda and my family members for their sustained support and encouragement. 


\title{
FORMULATION AND DEVELOPMENT OF PREBIOTIC CAPSULE
}

By

\section{FATHIMA HAJARA ASLAM}

\begin{abstract}
Felicitating probiotics by prebiotics attribute to harvest the promised health benefits from probiotics. This study aims to produce an integrated prebiotic combination which consists three different sources of plant extracts, which is capable to significantly increase the growth of two probiotic species, Lactobacillus acidophilus and Bifidobacterium animalis subsp. lactis BB-12 separately in vitro and thereby optimize health benefits. The three different sources of prebiotics considered in this experiment were fibre, polyphenolic extracts and non-digestible polysaccharide extracts which were isolated from Musa sp pseudostem, Sesbania grandiflora flowers and Artocarpus heterophyllus seeds. Eight treatments were designed by integrating three sources of prebiotics at two different levels i.e; fibre $(0.2 \%$ and $2 \%)$, polyphenolic extracts $(0.2 \%$ and $0.6 \%)$ and non-digestible polysaccharide extracts $(0.2 \%$ and $1.2 \%)$. Three treatments were designed by including only one ingredient at a time i.e; fibre $0.2 \%$, polyphenolic extracts $0.2 \%$ and non-digestible polysaccharide extracts $0.2 \%$. The effect of all eleven treatments were assessed against the growth promoting ability on L. acidophilus and $B$. animalis subsp. lactis-BB12 in liquid culture media and analysed using Minitab-16 Statistical software. The treatment which consisted $2 \%$ fibre, $0.2 \%$ polyphenolic extract and $0.2 \%$ non-digestible polysaccharide extract was able to produce a peak proliferation for $L$. acidophilus, while the treatment consisting $2 \%$ fibre, $0.6 \%$ polyphenolic extracts and $0.2 \%$ non-digestible polysaccharide extracts resulted in the highest proliferation for
\end{abstract}


B. animalis subsp. lactis BB-12 in vitro which were significantly different $(\mathrm{p}<0.05)$ than rest of the treatments. Test probiotic organisms supplemented with integrated prebiotic combinations, showed higher growth rate than, when grown with treatments with one prebiotic ingredient at a time. Musa sp fibre isolate showed higher potential to cause significant increase of L. acidophilus biomass, while polyphenolic extracts of Sesbania grandiflora flower imposed significant effect on increasing the growth of $B$. animalis subsp. lactis-BB12 when considered as separate entities in liquid culture media. The integrated treatments which showed the highest growth promoting effect on L.acidophilus and B.animalis subsp. lactis $\mathrm{BB}-12$ were encapsulated by hand filling to be administrated orally on daily basis. 